Original Article (short paper)

\title{
Resistance training improves the lipid profile, combat oxidative stress and inhibit MMP-2 activity in the left ventricle diet-induced obese rats
}

\author{
Anderson Diogo de Souza Lino ${ }^{1,2}$ (1) , Antônio Eduardo de Aquino Júnior ${ }^{1,3,4}$ (1) , Richard Diego Leite ${ }^{5}$ (1) , \\ Guilherme Fleury Fina Speretta ${ }^{6}$ (D) , Fernanda Dias de Moraes $^{7}$ (D) , Fernando Fabrizzi ${ }^{7}$ (D) , Gilberto Moraes ${ }^{7}$ (D) , \\ Heloisa Sobreiro Selistre-de-Araújo ${ }^{8}$ (i) , Ana Cláudia Garcia de Oliveira Duarte ${ }^{1}$ (i) \\ ${ }^{1}$ Universidade Federal de São Carlos, Centro de Ciências Biológicas e da Saúde, Departamento de Educação \\ Física e Motricidade Humana, Laboratório de Nutrição do Metabolismo Aplicado ao Exercício, São Carlos, SP, \\ Brasil; ' ${ }^{2}$ nniversidade Federal do Triangulo Mineiro, Programa de Pós-graduação em Educação Física, Uberaba, MG, \\ Brasil; ; ${ }^{3}$ Universidade de São Paulo, Grupo de Optica do Instituto de Física de São Carlos, IFSC, São Carlos, SP, \\ Brasil; ${ }^{4}$ Universidade Federal de São Carlos, Programa de Pós-graduação em Biotecnologia, São Carlos, SP, \\ Brasil; ${ }^{5}$ Universidade Federal do Espirito Santo; Centro de Educação Física E Desportos; Laboratório de Fisiologia do \\ Exercício (LAFEX), Vitoria, ES, Brasil; ${ }^{6}$ Universidade Federal de Santa Catarina, Centro de Ciências Biológicas, \\ Departamento de Ciências Fisiológicas, Florianópolis, SC, Brasil,; ${ }^{7}$ niversidade Federal de São Carlos, Centro de \\ Ciências Biológicas e da Saúde, Departamento de Genética, Laboratório de Bioquímica Adaptativa, São Carlos, SP, \\ Brasil;: ${ }^{8}$ Universidade Federal de São Carlos, Centro de Ciências Biológicas e da Saúde, Departamento de Ciências \\ Fisiológicas, Departamento de Genética e Evolução, Laboratório de Bioquímica e Biologia Molecular, São Carlos, SP, Brasil
}

\begin{abstract}
Aims: The purpose of the present study was to evaluate the effects of the resistance training (RT) on the lipid profile and metabolism, oxidative stress, and activity of metalloproteinase-2 (MMP-2) in the left ventricle (LV) of dietinduced obesity rats. Methods: Forty males Wistar rats 90 days-old were grouped into four groups (n=10): $i$ ) Sedentary group (SED); ii) Obese sedentary group, feed with high-fat diet (Ob-SED); iii) Resistance Trained group (RT), and iv) Obese Resistance trained group (Ob-RT). The LV was assayed to Obesity index, LV lipid content, citrate synthase activity, lipid peroxidation (TBARS), enzymatic and non-enzymatic antioxidant systems, lipid profile, cardio-metabolic parameters, and activity of MMP-2. Results: High-fat diet was associated with manifestations of the obesity, body mass gain, and increased obesity index, accompanied by an alteration in the lipid profile. On the other hand, RT was able to prevent body weight gain, to reduce the obesity index and to improve the lipid profile, to elevate the activation of the citrate synthase, and to decrease MMP-2 activity in the LV of obese rats. Conclusion: RT positively modulated blood lipid profile and antioxidant enzymes preventing the increased activity of MMP-2 in the left ventricle from rats fed with high-fat diet.
\end{abstract}

Keywords: high-fat diet; resistance training; lipid profile; obesity; matrix metalloproteinase 2; oxidative stress.

\section{Introduction}

Obesity, a multifactorial disease linked to a complex relationship among biological, psychosocial, and behavioral factors, likes a lifestyle ${ }^{1}$. It has been qualified as the epidemic of this century and $95 \%$ of the cases come from an exogenous origin. Daily intakes of excessive calories associated with low physical activity are the main cause of many exogenous obesity cases in adults ${ }^{2,3}$. Obesity is one of the major risk factors for cardiac diseases such as heart failure ${ }^{4}$, ischemic heart disease ${ }^{5,6}$, as well as metabolic diseases insulin resistance, and dyslipidemia ${ }^{6}$. Besides, obesity is associated with increased myocardium lipid peroxidation and increased susceptibility to oxidative damage, a process that starts with the metabolic increase of reactive species of oxygen (ROS) and nitrogen $(\mathrm{RNS})^{7,8}$.

To protect from the harmful effects of the use of $\mathrm{O}_{2}$ in the cellular respiration process, the body has a complex antioxidant defense system, which is composed of various enzymatic and non-enzymatic antioxidants. The main antioxidant enzymes are the superoxide dismutase (SOD), catalase (CAT), and glutathione peroxidase (GPx), which convert the oxidizing agents into non-toxic molecules ${ }^{8}$. Obesity-induced by a high-fat diet generates oxidative stress in the heart and other tissues, mostly due to a decrease in the activity of antioxidant enzymes.

Some molecules are associated with the remodeling of cardiac tissue in obesity and oxidative stress. One of these molecules is matrix metalloproteinases (MMPs). MMPs are a member of the family of endopeptidases zinc-dependent. These molecules contribute to regulating normal cardiac tissue remodeling. Among them, MMP-2 is a key endopeptidase involved in the remodeling of the cardiac extracellular matrix ${ }^{10,11}$. Obesity, hypertension, dyslipidemia, and oxidative stress are possibly related to left ventricular (LV) remodeling. Thus, factors mentioned above are considered to stimulate the synthesis and activity of MMP-2, which can lead to pathological remodeling of the LV in obese individuals ${ }^{10-12}$.

The activation of MMP- 2 by oxidative stress due to the condition of obesity occurs through the phosphorylation-dephosphorylation 
of this enzyme (MMP-2) $)^{13}$. In this sense, these reactions can be carried out either by proteinases ${ }^{14,15}$ or by post-translational modifications of the $72-\mathrm{kDa}$ of the MMP-2 zymogen (proMMP-2) by S glutathiolation and nitrosylation. Furthermore, in an obesity condition, MMP-2 can be activated directly by nitric peroxide $\left(\mathrm{ONOO}^{-}\right)$. This process occurs through a non-proteolytic mechanism that involves the glutathiolation $\mathrm{S}$ of the pro-peptide group cysteine sulfhydryl in a reaction that requires small concentrations of $\mathrm{ONOO}^{-}$at the equimolar and intracellular levels of reduced glutathione ${ }^{10,16}$.

To avoid the deleterious effect of obesity on the LV remodeling, there is a growing interest in the resistance training (RT) as an exercise modality capable of regulating the activity of proteins associated with cardiac remodeling (MMPs) ${ }^{10,11,17}$. Previous studies have shown that the RT results in a cardioprotective effect ${ }^{18-20}$. This effect may be due to the improvement of the antioxidant system and consequent reduction of oxidative stress, regulating the activity of MMP-2, and promoting an improvement in cardiac functions. However, little is known if an RT protocol can prevent the possible negative changes mentioned above about the LV in rats fed a high-fat $\operatorname{diet}^{18}$.

Thus, the present study aims to test the hypothesis that the RT can prevent the increase of the adiposity index, to modulate the lipid profile, to inhibit oxidative stress, and to balance the activity of MMP-2 in the LV of Wistar male rats with induced obesity from feeding with high-fat diet.

\section{Methods}

\section{Animal Care}

The experimental protocol was approved by the Committee of Experimental Animals from the Federal University of São Carlos/UFSCar (Protocol \# 31/2009). All experimental procedures were according to the principles outlined by the Brazilian College of Animal Experimentation (Colégio Brasileiro de Experimentação Animal - COBEA), and Guide for the Care and Use of Laboratory Animals, published by the US National Institute of Health (NIH, $8^{\text {th }}$ Edition, 2011).

Forty males of Wistar rats (Rattus novergicus var. Albinus, Rodentia, Mammalia) 30 days-old from a breeding colony of the Federal University of São Carlos (UFSCar), SP, Brazil. For acclimatization, the animals were held in collective polypropylene cages $(\mathrm{n}=5)$, kept in a controlled constant temperature $\left(22 \pm 2^{\circ} \mathrm{C}\right)$, and $12-12 \mathrm{~h}$ light-dark cycle. During the acclimation period, the rats received standard rat chow (MP-77; Primor ${ }^{\circledR}$, São Paulo, Brazil) and tap water ad libitum. Daily, body mass (BM) and food intake were weighed.

\section{Experimental Design}

Upon reaching 90 days, the animals were randomly distributed into four experimental groups $(\mathrm{n}=10)$. Each animal was condition one per polypropylene cages (30x20x13 cm): $i$ )
Eutrophic sedentary group, fed with standard rats' chow (SED); ii) obese sedentary group, fed with high-fat diet (Ob-SED); iii) eutrophic trained group, fed with standard rats' chow and submitted to Resistance Training protocol (RT); and $i v$ ) obese trained group, fed with high-fat diet and submitted to Resistance Training protocol (Ob-RT).

The study was divided into two steps. The first one was conducted by three weeks for obesity induction. After this period, the second stage began, which consisted of maintaining the supply of the high-fat diet for the animals of the obese groups for another eight weeks and performing the protocol of resistance training in the animals of the trained groups, indicated above, illustrated in figure 1.

\section{Standard and high-fat diet}

The eutrophic rats (SED and RT groups) were fed with standard rat chow pellets (MP-77; Primor ${ }^{\circledR}$, São Paulo, Brazil). Animals assigned to the obese groups (Ob-SED and Ob-RT) were fed with the high-fat diet as reported in the experimental protocols, with an amount of $20 \%$ fat. The composition of both diets is expressed in table 1. The high-fat diet was performed with a mixture of standard rat chow plus peanuts, milk chocolate, and sweet biscuits to reach the wanted nutrient composition and a palatable diet ${ }^{21}$. The high-fat diet, after ready to be offered, was pelletized to the same size as the rat chow diets (Table 1). All ingredients were weighed and mixed according to laboratory procedures. The constitution of diets, normal and high-fat, was carried out by bromatology [CBO laboratory analysis (https:// www.labcbo.com)].

\section{Obesity-induced period}

To induce obese conditions, the rats designed to the obese groups were fed with the high-fat diet for three weeks ${ }^{21}$.

\section{Resistance training protocol (RT)}

The RT protocol was performed for eight weeks after the obesity-induced period. The protocol consisted of 8-12 dynamic movements (reps) per climb, as previously described ${ }^{22}$ and posteriorly adapted ${ }^{23,24}$.

The rats were adapted to RT protocol for two days interlarded by rest periods of $72 \mathrm{~h}$ to totalize eight weeks. During the training, the rats had to climb a vertical ladder with a weight attached to the animal tail. Initially, the rats were familiarized with the training procedure by climbing the leader with an object mimicking the weight and progressively heavier ( $75 \%$ of the body mass followed by the addition of $30 \mathrm{~g}$ toward the "maximum carrying capacity"). That step allowed evaluating the maximum load for each animal (one point before the load being impossible to be successfully carried over the leader end to end). Failure was determined when the animal could not progress up the ladder after three successive stimuli. RT session consisted of 4 ladder climbs with $50 \%, 75 \%, 90 \%$, and $100 \%$ of the previously determined maximum-carrying-capacity to each rat. If 
the rat reached $100 \%$ of the carrying load, an additional $30 \mathrm{~g}$ were added until a new maximum-carrying-capacity with a fifth ladder extra climb. The rest period between each climb was 120 s.

\section{Volume of carrying the load}

Load Total Volume (LTV) was determined as the sum of every Load Volume per climb per animal performed until exhaustion (LVi) in each session and expressed as:

$$
\mathrm{LTV}=\sum_{1}^{n} L V \mathrm{i}
$$

\section{Euthanasia and tissue collection}

Twenty-four hours before euthanasia, all animals were weighed. Animals were euthanized by decapitation $48 \mathrm{~h}$ after the last training session and blood samples were immediately collect and centrifuged at $10.000 \mathrm{x}$ g for $10 \mathrm{~min}$. Before euthanasia, the rats were in eight hours of fasting. Supernatant plasma was separated and stored for posterior analyses. The hearts were collected, rinsed with cold saline, and weighted. The left ventricles (LVs), including the septum, were isolated, weighed, placed in cryogenic tubes, dipped into liquid nitrogen, and stored at $-80^{\circ} \mathrm{C}$ for posterior analyses.

\section{Visceral Fat Depots (Pads)}

The Mesenteric, Urogenital, and Retroperitoneal fat deposits were excised and immediately weighed to avoid weight loss. The mesenteric fat pad consisted of adipose tissue surrounding the gastrointestinal tract from the gastroesophageal sphincter to the end of the rectum, and special care was taken in distinguishing and removing pancreatic cells. The urogenital fat pad included adipose tissue surrounding the kidneys, ureters, and bladder. The retroperitoneal fat pad was taken as distinct deposits behind each kidney along the lumbar muscles ${ }^{25}$.

\section{Adiposity Index}

Adiposity index (AI) is presented as the sum of the intra-abdominal adipose tissue mass and subcutaneous fat deposits (FD) divided by the total body mass (BM) and expressed as percentage accordant with the expression ${ }^{26}$ :

$$
A I(\%)=\left(\frac{\sum F D}{B M}\right) \cdot 100
$$

\section{Biochemical Parameters}

Glycaemia was determined by glucose-oxidase; total triacylglycerol (TGL), total cholesterol (TC), and high-density lipoprotein cholesterol (HDL-c) were assayed enzymatically with specific lab kits (Laborlab, Sao Paulo, Brazil). The test was performed in duplicate and the coefficient of variation was $p<0.05$. Serum very-low-density lipoprotein (VLDL-c) and low-density lipoprotein cholesterol (LDL-c) concentrations were calculated with the Friedewald equation ${ }^{27}$, and atherogenic indices were calculated as follow: a- LDL-c $(\mathrm{mg} / \mathrm{dL})=$ Total cholesterol $-($ HDL-c - TGL / 5); b- VLDL-c = Triglycerides/5; c- Castelli's Risk Index I = Total cholesterol/HDL-C; d- Castelli's Risk Index II = LDL-c/HDL-c; e- TGL/HDL-c ratio ${ }^{28}$.

\section{Colorimetric Assays}

All samples of each enzyme were assayed on the same day. To determine the specific enzymatic activities, $25 \mathrm{mg}$ of LV samples were used to perform the cell extracts. Briefly, tissues were homogenized in $1.0 \mathrm{~mL}$ of $0.1 \mathrm{M}$ potassium phosphate buffer $\mathrm{pH} 7.0$ plus $0.25 \mathrm{M}$ sucrose $(1: 1)$ and centrifuged at $20.000 \mathrm{xg}$ for $5 \mathrm{~min}$ at $5^{\circ} \mathrm{C}$. The supernatants cell extracts were used as an enzyme source. The enzyme's activities were assayed following the rules specified in the Cayman's assay kit. Total-SOD and mitochondrial Superoxide Dismutase (Mn-SOD) was read at 440nm, Catalase (CAT) was read at 540nm, and Lipid Peroxidation (TBARS) was read at 540nm. Approximately $50 \mu \mathrm{g}$ of protein from the extract was used per enzyme assayed. Glutathione Peroxidase (GPx) and Glutathione Oxidase (GSH) were assayed according to Beutler ${ }^{29}$. Shortly, GPx was assayed with $100 \mu \mathrm{g}$ of protein from LV extracts (as reported above) per enzyme assay. Enzyme activity was read at 340nm. Glutathione Oxidase GSH was determined in LV tissue extracts made under an ice bath in Na-phosphate buffer 0.2M pH 7.0, to 50mg of LV tissue. The optical density was read at $412 \mathrm{~nm}$. Citrate Synthase activity

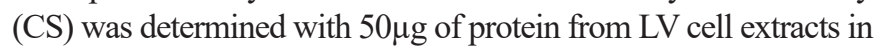
$100 \mathrm{mM}$ Tris-HCL buffer pH 7.5 and read at 412nm (CS Assay Kit CS0720-Sigma). Protein concentrations in the homogenates of LV samples were determined with Bradford reagent against a standard solution of bovine serum albumin (BSA-Sigma) and read at $540 \mathrm{~nm}^{30}$.

\section{Determination of total Left Ventricle lipids content}

The total lipid concentration was determined by the colorimetric method sulfo-phospho-vanillin, adapted to tissues. An aliquot of $50 \mathrm{mg}$ LV was used for each animal to determine the lipid concentration ${ }^{31}$. The OD was performed at $540 \mathrm{~nm}$. All samples were assayed on the same day.

\section{MMP-2 Activity by Gelatin Zymography}

An aliquot of $50 \mathrm{mg}$ of frozen LV was rinsed 3 times with cold saline, incubated at $4^{\circ} \mathrm{C}$ for $24 \mathrm{~h}$ in extraction buffer ( $10 \mathrm{mM}$ cacodylic acid pH 5.0; $0.15 \mathrm{M} \mathrm{NaCl} ; 1 \mu \mathrm{M} \mathrm{ZnCl} 2 ; 20 \mathrm{mM} \mathrm{CaCl} 2 ; 1.5 \mathrm{mM}$ NaN3; $0.01 \%$ Triton X-100 [v/v]) at a ratio of $25 \mu \mathrm{L}$ of buffer per $\mathrm{mg}$ of tissue, and centrifuged for $20 \mathrm{~min}$ at $13.000 \mathrm{x}$ in cold-bath at $4^{\circ} \mathrm{C}$. Aliquots containing $30 \mu \mathrm{g}$ of total protein, determined by BCA Protein Assay Kit (Pierce ${ }^{\circledR}$, Rockford, IL, USA), were applied in the lanes of sodium dodecyl sulfate (SDS)-10\% polyacrylamide gels prepared with $1 \mathrm{mg} \mathrm{mL}^{-1}$ of gelatin. After electrophoresis, the gels were washed twice for $20 \mathrm{~min}$ in $2.5 \%$ Triton X-100 to remove 
the SDS. Gels were rinsed and incubated in buffer substrate $(50 \mathrm{mM}$ Tris- $\mathrm{HCl} \mathrm{pH} 8.0 ; 5 \mathrm{mM} \mathrm{CaCl} 2 ; 0.02 \% \mathrm{NaN} 3$ ) at $37^{\circ} \mathrm{C}$ for $20 \mathrm{~h}$ and were stained with Coomassie Brilliant Blue for $1 \mathrm{~h}$ and distained with acetic acid: methanol: water (1:4:5) for visualization of the activity bands. Negative control was performed with EDTA in the gel-loaded samples and incubation buffer.

The gels were photographed with a Canon G6 Power Shot 7.1 megapixels camera. The images were processed with Irfan View for Microsoft Windows. All images were fitted to reach the same brightness and contrast. Densitometry of the MMP-2 protein bands was performed with the Gene Tools version 3.06 software (Syngene, Cambridge, UK).

\section{Statistics}

Results were expressed as mean \pm standard error of the mean (S.E.M). The normality of data was evaluated by the Kolmogorov-Smirnov and the homoscedasticity was assessed by Levene's test. Two-way analysis of variance was used to test the effects of training, high-fat diet, and their interactions, followed by the Post-Hoc-Bonferroni $(\mathrm{p}<0.05)$. The Student's t-test $(\mathrm{p}<0.05)$ was used to compare the volume of carrying load between the groups RT $v s$ Ob-RT. The IBM SPSS ${ }^{\circledR}$ Statistics software version 20 for Macintosh was used to evaluate the present data.

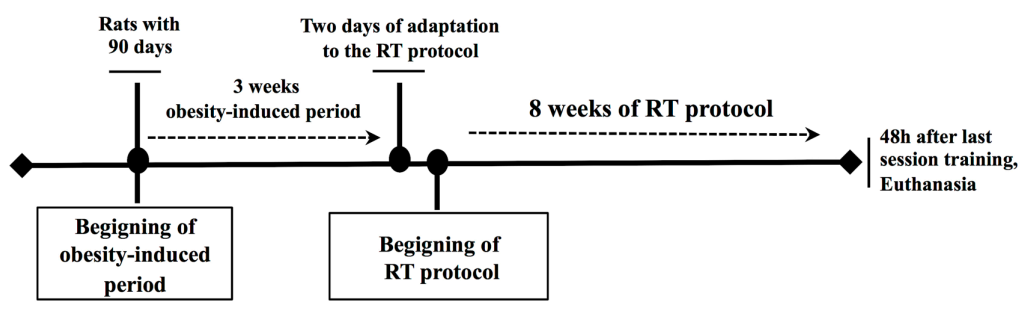

Figure 1 - Representation of the experimental design of the study.

Table 1 - Composition of the standard- and high-fat diet.

\begin{tabular}{|c|c|c|}
\hline & Standard & High-fat diet \\
\hline & \multicolumn{2}{|c|}{$\%$} \\
\hline Protein & 22.81 & 18.12 \\
\hline Fat & 4.80 & 20.00 \\
\hline CHO & 39.23 & 32.90 \\
\hline Fiber & 5.82 & 2.97 \\
\hline MM & 6.87 & 3.29 \\
\hline Humidity & 12.47 & 14.72 \\
\hline$*$ Kcal g ${ }^{-1}$ & 3,854 & 4,665 \\
\hline
\end{tabular}

The composition of Standard- and high-fat diet are expressed in percentage (\%) per 100g of diet. CHO, Carbohydrate; MM, mineral material. *The caloric content of the diet was determined with an adiabatic calorimeter IKA-500 and expressed in Kcal.

\section{Results}

\section{Body mass (BM) evolution}

The initial BM of all groups was nearly the same indicating weight homogeneity among the samples at the zero point (Figure 2A). After the third experimental weeks, the Ob-SED group fed with a high-fat diet depicted increased BM in comparison with all others $(p<0.03)$. This data despite being observed from the end of the first experimental week was significant from that point and pronounced from it through the whole experimental span. After the beginning of the experimental period for training, the RT avoided the BM gain in the Ob-TR throughout the experimental period, compared to the Ob-SED $(\mathrm{p}<0.05)$.

The Adiposity index is illustrated in figure $2 \mathrm{~B}$. The rats from the $\mathrm{Ob}-\mathrm{SED}$ group showed a higher Adiposity index than those from the SED group $(p<0.001)$. The RT group rats did not differ from the SED group ones but showed lower percentage values as compared with Ob-SED group rats $(\mathrm{p}<0.001)$ and with RT group rats $(\mathrm{p}<0.001)$. However, eight weeks of RT resulted in none effects on the Adiposity index in obese trained animals as compared with obese sedentary ones (Ob-RT vs Ob-SED). 


\section{Heart and left ventricle (LV) mass}

Neither high-fat diet nor RT promoted any change in the heart and LV mass of the groups.

\section{Evolution of total carrying load volume (ETCL)}

There was no difference between the RT and Ob-RT in the ETCL volume over the whole experimental period (Figure 3).

\section{Glycaemia and Plasma Lipid Profile}

Glycaemia of obese rats submitted to RT (Ob-RT) was lower than that from all the other groups $(p<0.001)$, which were similar among them (Figure 4A).

The values of total cholesterol (Figure 4B) were lower in the rats from both trained groups (RT and Ob-RT) and those in the $\mathrm{Ob}$ SED group ( $\mathrm{p}<0.03, \mathrm{p}<0.02$, respectively). In the $\mathrm{Ob}$-SED group, the serum triglycerides (TGL) of the rats was higher than that of the SED group animals $(p<0.03)$. The exercise training also reduced the TGL concentration in the rats of the RT $(\mathrm{p}<0.002)$ and Ob-RT groups $(p<0.002)$ when Ob-SED animals are used for comparison (Figure 4C). The concentration of HDL-c rats was higher than that observed in the animals from the $\operatorname{SAD}(\mathrm{p}<0.001)$ and $\mathrm{Ob}-\mathrm{SED}$ groups $(\mathrm{p}<0.001)$. A positive effect of the RT was observed in Ob-RT animals concerning the SED group $(\mathrm{p}<0.02)$ and $\mathrm{Ob}-\mathrm{SED}(\mathrm{p}<0.003)$ rats. Resistive training led to similar concentrations of HDL-c between Ob-RT and RT groups (Figure 4D). Concentrations of LDL-c were similar among all groups (Figure 4E). The condition of obesity without exercise in the rats from the SED group elevated the VLDL-c concentration $(p<0.04)$ but, among the rats from RT condition and those from the SED group any difference was not observed.

\section{Cardio-metabolic Parameters}

Comparisons among all groups showed that the cardio-metabolic risk was attenuated by RT protocols in both groups RT and Ob-RT. In the RT group Castelli's ratio I was decreased as compared either with SED $(p<0.003)$ or Ob-SED ones $(p<0.001)$. The Ob-RT group rats presented no significant difference values in comparison with the SED $(p<0.007)$ and Ob-SED ones $(p<0.001)$ (Figure 5A). A decrease in the Castelli's ratio II (LDL-c/HDL-c) was observed in both RT and Ob-RT groups as compared with Ob-SED $(p<0.001)$. Regarding Castelli's ratio III, we observed that the RT group showed a decreased ratio when compared to the SED group $(p<0.03)$. The $\mathrm{Ob}-\mathrm{SED}$ increased the ratio when compared to $\operatorname{SED}(\mathrm{p}<0.02$, Figure 5B). Regarding, the RT, even to the Ob-RT group, showed lower values for the Castelli's ratio III when compared to the Ob-SED group ( $\mathrm{p}<0.001$, for both. Figure $6 \mathrm{C}$ ).

\section{Total lipid content and the citrate synthase activity (CS)}

The Ob-RT group showed higher total lipid content in the LV $(\mathrm{p}<0.05)$ when compared to the RT group RT. No change was observed between the other groups. The specific activity of LV CS increased in the Ob-RT group when compared to the other groups $(\mathrm{p}<0.004)$. The rats from Ob-SED and RT groups depicted the same CS activity of the SED group (Figure 6).

\section{Lipid peroxidation (TBARS) and Antioxidant system}

Lipid peroxidation (TBARS) in LV tissue of rats submitted to RT protocol was increased in comparison with the other groups $(p<0.05)$. None difference was observed between the SED groups and the Ob-RT group. These data are shown in table 2 . The values of Total-SOD activity in the RT group were higher than those of SED and Ob-SED groups $(\mathrm{p}<0.001)$. In obese and trained animals (ObRT) that value was lower than that in the RT group $(\mathrm{p}<0.001)$. The Mn-SOD activity was higher in rats of the RT group in contrast to those from the SED group $(p<0.03)$. The activity of CAT increased in the animals submitted to the RT protocol in comparison with those from Ob-SED ones $(p<0.05)$. Comparison between obese trained (Ob-RT) and obese sedentary (Ob-SED) animals depicted an increase in CAT activity in the first ones $(\mathrm{p}<0.001)$. The RT protocol, in both eutrophic and obese groups (RT and Ob-RT), was able to lessen the GHS activity as compared with the Ob-SED group ( $<<0.002$ and $\mathrm{p}<0.01$, respectively). The rats from the RT group compared with those from SED, Ob-SED and Ob-RT ones boost the SOD/GPx ratio values $(\mathrm{p}<0.001)$. Similar results were observed concerning the SOD/CAT ratio. There was no significant change of specific activities between SED groups neither to Total-SOD, Mn-SOD, GPx, CAT and GSH nor to the ratio SOD/GPx and SOD/CAT (Table 3).

\section{MMP-2 Activity by Gelatin Zymography}

The activity expression of the Pro Isoform of MMP-2 did not change in Ob-SED rats as compared with those from the SED group. This isoform reached the highest expression in RT rats, and the ObRT animals depicted intermediate values (Figure 7-2A). The MMP-2 Intermediate Isoform expression was maximum in Ob-RT rats and minimum in SED and Ob-SED rats, while in RT animal this expression was intermediate $(\mathrm{p}<0.05)$. In the Active Isoform, the highest expression was seen in Ob-SED rats and the others remained equal $(\mathrm{p}<0.05)$ (Figure 7-2C).

Table 2 - Rat Heart weight and LV mass of Sedentary (SED), Obese sedentary (Ob-SED), resistance training (RT), and obese trained groups.

\begin{tabular}{lcccc}
\hline & SED & Ob-SED & RT & Ob-RT \\
\hline Heart $(\mathrm{g})$ & $1.94 \pm 0,16$ & $1.89 \pm 0.10$ & $1.87 \pm 0.08$ & $1.74 \pm 0.08$ \\
$\mathbf{L V}(\mathrm{mg})$ & $0.75 \pm 0,03$ & $0.75 \pm 0.01$ & $0.71 \pm 0.01$ & $0.75 \pm 0.02$ \\
\hline
\end{tabular}


Table 3 - Biochemical assays of antioxidant activity and lipid peroxidation in the rat LV from Sedentary (SED), Obese sedentary (Ob-SED), resistance training $(\mathrm{RT})$, and obese trained (Ob-RT) groups.

\begin{tabular}{|c|c|c|c|c|}
\hline & SED & Ob-SED & RT & Ob-RT \\
\hline Total-SOD (nmol/min/mg) & $11.27 \pm 0.78$ & $10.91 \pm 0.51$ & $25.49 \pm 1.33^{\mathrm{a}, \mathrm{b}}$ & $12.36 \pm 0.86^{\mathrm{c}}$ \\
\hline Mn-SOD (nmol/min/mg) & $1.52 \pm 0.15$ & $1.76 \pm 0.18$ & $2.19 \pm 0.18^{\mathrm{a}}$ & $2.81 \pm 0.14^{\mathrm{a}, \mathrm{b}, \mathrm{c}}$ \\
\hline $\begin{array}{l}\text { CAT } \\
\left(\mathrm{nmol} / \mathrm{min} \mathbf{m g}^{-1}\right)\end{array}$ & $2.96 \pm 0.13$ & $2.51 \pm 0.08$ & $3.08 \pm 0.16^{\mathrm{b}}$ & $3.45 \pm 0.19^{b}$ \\
\hline $\begin{array}{l}\text { GPx } \\
(\mathrm{nmol} / \mathrm{min} / \mathrm{mg})\end{array}$ & $72.14 \pm 2.81$ & $72.09 \pm 2.81$ & $73.42 \pm 7.49$ & $73.85 \pm 4.44$ \\
\hline $\begin{array}{l}\text { GSH } \\
(\mathrm{nmol} / \mathrm{g})\end{array}$ & $1,234 \pm 42$ & $1,385 \pm 57$ & $1,100 \pm 53^{b}$ & $1,148 \pm 46^{\mathrm{b}}$ \\
\hline $\begin{array}{l}\text { TBARS } \\
(\mu M)\end{array}$ & $18.51 \pm 0.77$ & $17.30 \pm 2.23$ & $23.72 \pm 2.31^{\mathrm{b}}$ & $15.65 \pm 0.97^{c}$ \\
\hline SOD/GPx & $0.17 \pm 0.01$ & $0.14 \pm 0.01$ & $0.36 \pm 0.04^{\mathrm{a}, \mathrm{b}}$ & $0.16 \pm 0.01^{\mathrm{c}}$ \\
\hline SOD/CAT & $4.01 \pm 0.42$ & $4.44 \pm 0.26$ & $8.67 \pm 0.72^{a, b}$ & $3.70 \pm 0.47^{\mathrm{c}}$ \\
\hline
\end{tabular}

The letter superscript represents statistical difference between groups by two-way ANOVA followed by the Post-Hoc-Bonferroni test $(\mathrm{p}<0.05)$.

$\mathrm{SED} \neq \mathrm{a}$

$\mathrm{Ob}-\mathrm{SED} \neq \mathrm{b}$

$\mathrm{RT} \neq \mathrm{c}$
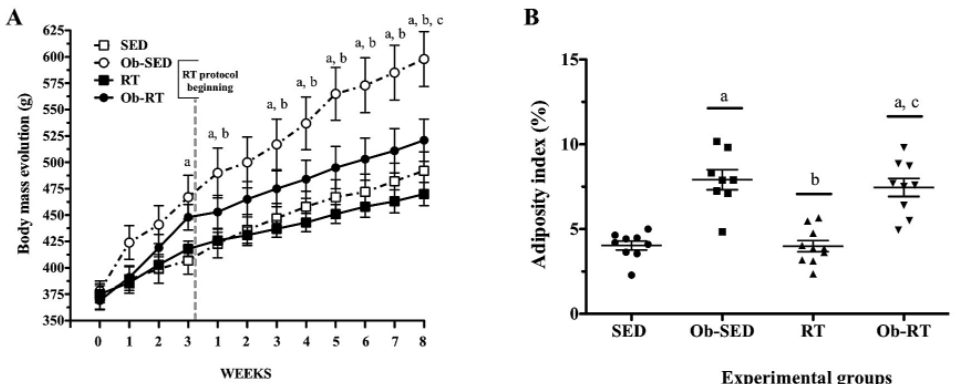

Figure 2 - Effect of high-fat diet and RT on A) body mass evolution (g); and B) Adiposity index (\%).

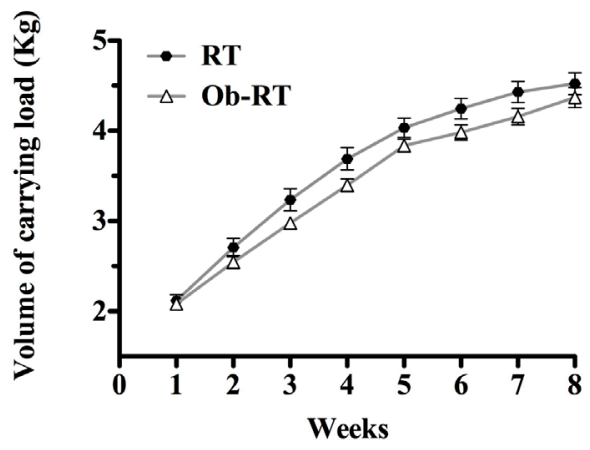

Figure 3 - The volume of the carrying load $(\mathrm{Kg})$. RT, resistance training group; and Ob-RT, obese trained group. 


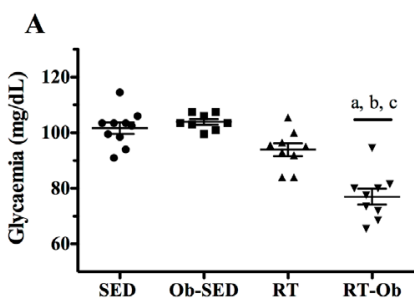

B
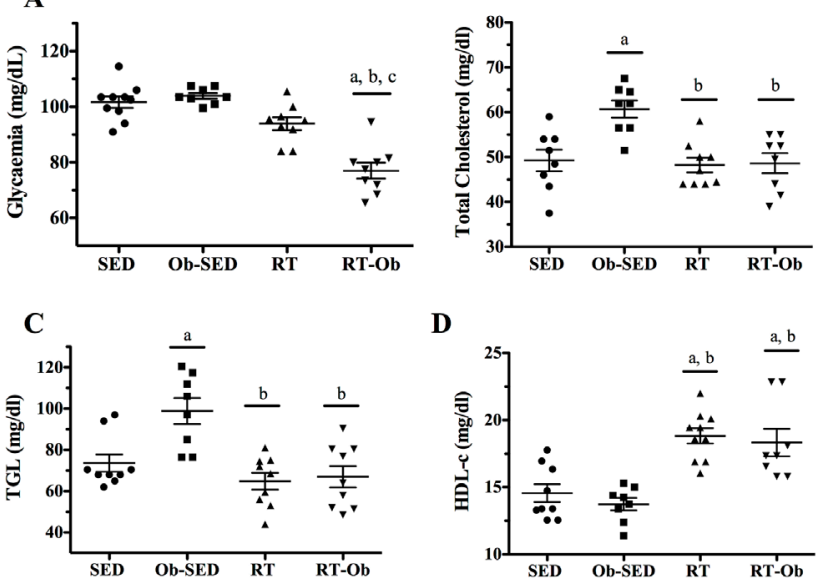

D
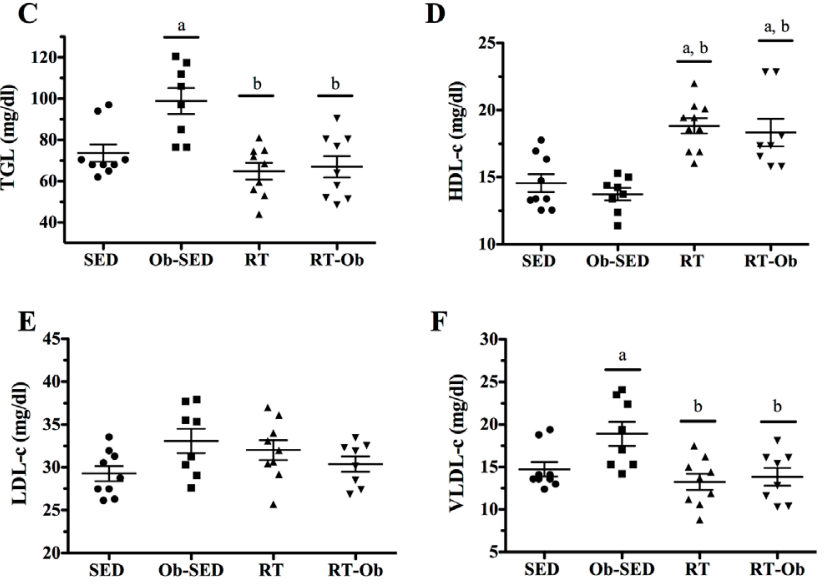

Figure 4 - Effect of high-fat diet and RT on serum: A) Glycaemia; B) Total Cholesterol; C) Triglycerides (TGL); D) High-density lipoprotein (HDL); E) Lowdensity lipoprotein and; F) Very low-density lipoprotein (VLDL), in mg/dL. SED, Sedentary group; Ob-SED, Obese sedentary group; RT, resistance training group; and Ob-RT, obese trained group.

A

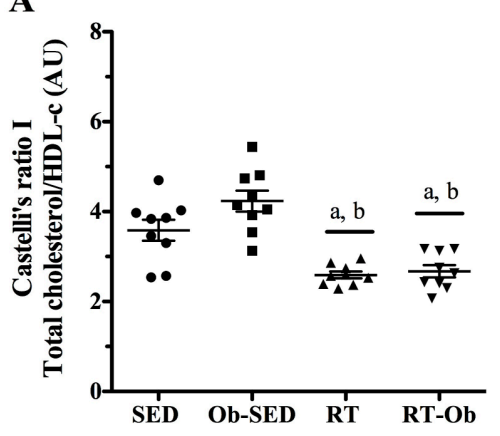

B
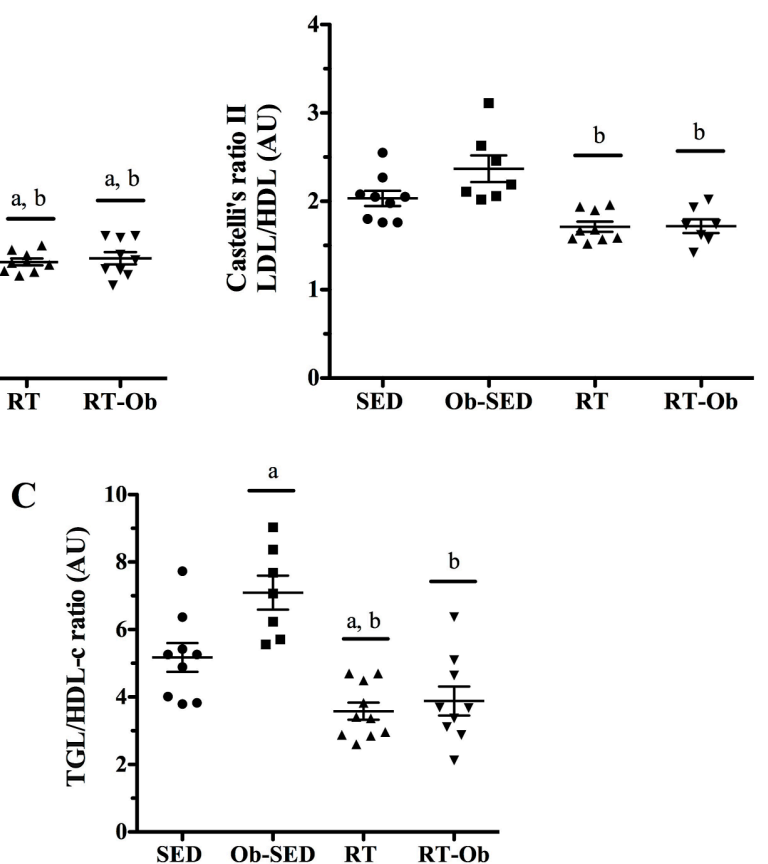

Figure 5 - Effect of high-fat diet and RT on cardio-metabolic parameters. In Panel A- Castelli’s ratio I, Total cholesterol/HDL-c; B- Castelli’s ratio II LDL-c/ HDL-c; and C- LDL-c: Low-density lipoprotein cholesterol; HDL-c: high-density lipoprotein cholesterol. SED, Sedentary group; Ob-SED, Obese sedentary group; RT, resistance training group; and Ob-RT, obese trained group. 
A

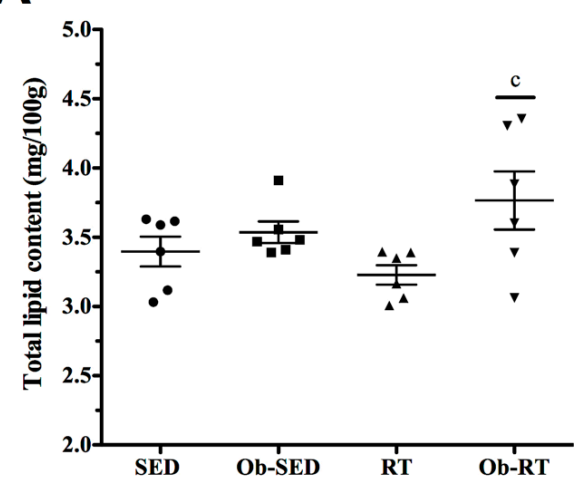

B

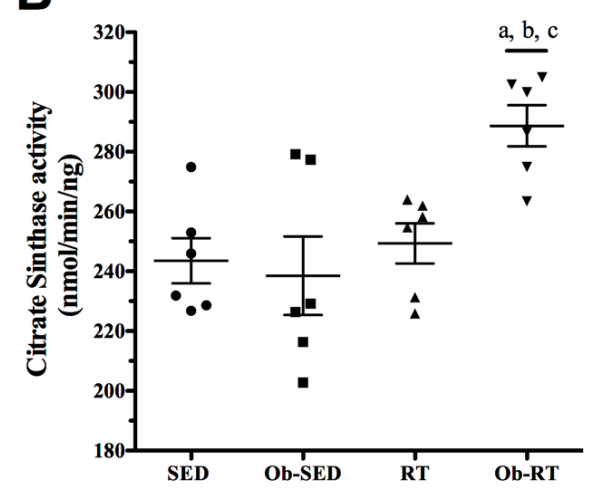

Figure 6 - Effect of high-fat diet and RT in A- total lipid content (mg/100g) and B- citrate synthase activity (nmol/min/ng). SED, Sedentary group; Ob-SED, Obese sedentary group; RT, resistance training group; and Ob-RT, obese trained group.

1

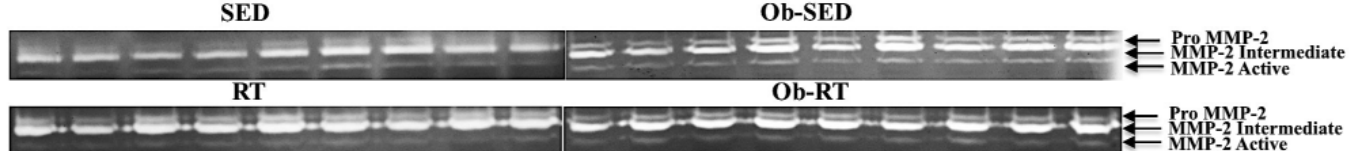

2

A

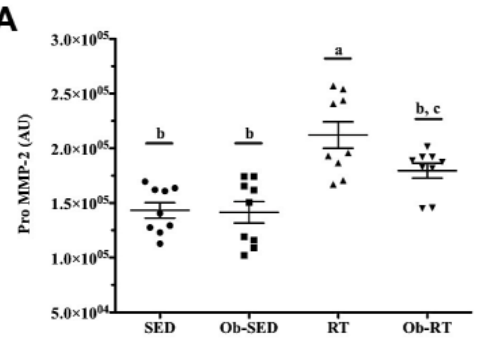

B

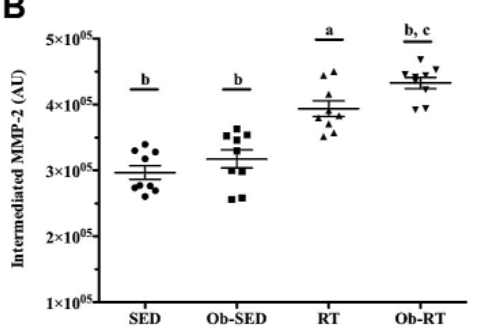

C

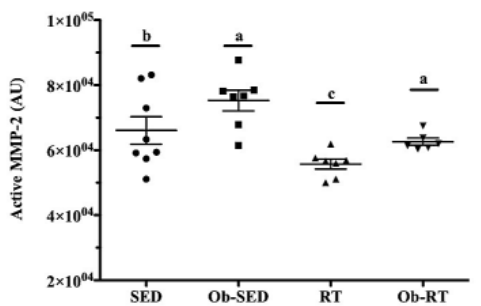

Figure 7 - Effect of high-fat diet and RT on isoforms' activity of matrix metalloproteinases 2 (MMP-2) in the left ventricle (LV). SED, Sedentary group; ObSED, Obese sedentary group; RT, resistance training group; and Ob-RT, obese trained group.

\section{Discussion}

The extra energy surplus from a high-fat diet combined with a sedentary lifestyle and other factors result in increased adipose tissue mass, which characterizes obesity, among other factors. Obesity is a multifactorial disease basically from dietary unbalance. Consuming of a high-fat diet is associated with significant increase adiposity, ectopic fat accumulation, altered blood lipid profile and cardiac abnormalities in rats ${ }^{32-35}$, and humans ${ }^{36}$. Also, abnormal alterations in the cardiac structure and function are associated with the oxidative stress and activity of proteolytic enzymes such as MMP- $2^{10}$.

The present study showed that the RT was an important strategy to positively modulating the blood lipid profile, antioxidant enzymes as Total-SOD, Mn-SOD, and GSH. Even that, RT was able to prevent the increased activity of MMP- 2 in the left ventricular in obese rats induced by diet.
In previous studies, our group has shown that the palatable high-fat diet is recommended as a good strategy to induce obesity in rats. This diet leads to increased BM and results in deleterious effects related to obesity, such as an increase of hepatic fat content (NAFLD) and alteration of serum lipid profile ${ }^{32,34}$, an increase of adipocyte area followed by metabolic chronic pro-inflammatory state ${ }^{24}$. In the present study, eight weeks of a high-fat diet was able to promote higher BM, but RT prevented elevation of BM gain, in Wistar rats. Curiously, in the course of RT protocol development, obese animals presented equal volumes of carrying the load. However, whether those volumes were relativized by a ratio with BM, obese animals showed a lower relative volume of workload compared with eutrophic animals. Besides, obesity can cause a reduction in muscle strength concerning body size. On the other hand, physical exercise is associated with an improvement in muscle strength ${ }^{37}$.

In a report on possible differences in the peripheral strength 
of women, no differences are observed in obese versus lean subjects for absolute isokinetic strength in knee extension and isometric handgrip. However, when correcting the values for fat-free mass, the muscle strength is at least $6 \%$ lower in obese women compared with lean ones ${ }^{38}$. Obese men also depict higher muscle power and torque ${ }^{39}$. However, whether relativized by $\mathrm{BM}$, the obese men showed a reduction of the relative muscle power and torque in comparison with lean ones. Our results are showing that obese rats present equal absolute capacity to perform the training, but obesity leads to a training decline capacity when it is relativized by the ratio of the BM to every animal. Even that the RT has avoided BM gain in animals fed with a high-fat diet it did not show positive effects in reducing the adiposity index values. The RT has shown to be an important tool for increasing the strength, and it is still very efficient to reduce body fat ${ }^{40}$. Besides, RT is an important tool against the negative effects of obesity, such as elevated levels of glycemia ${ }^{41}$.

Obesity can elevate blood glucose levels. In our study, the RT may lead to decrease blood glucose levels in obese animals. The practice of RT by obese shifts the predominance of lipids as the energy source to carbohydrates, and signalizes to increasing of glucose uptake ${ }^{41}$. It occurs by elevating the expression and the degree of phosphorylation / activity of isoform insulin receptor substrate 1 and 2 (IRS-1, IRS-2), phosphatidylinositol-3-kinase $(\mathrm{PI} 3 \mathrm{~K})$ and protein kinase $\mathrm{B}^{42}$. It results in an increase in glucose transporters (GLUT4) to the plasma membrane ${ }^{43,44}$. A study that investigated the effects of 12 weeks of RT in obese rats showed that there was greater phosphorylation/activity of Akt stimulated by insulin and an increase in GLUT4 expression in skeletal muscle fibers, contributing to the reduction of glycemia ${ }^{45}$.

In addition, RT can stimulate glucose uptake-synlization pathways independent of insulin. One of these pathways is stimulated involving a key enzyme activated by muscle contraction, called AMP-activated protein kinase (AMPK) ${ }^{46}$. The increase in glucose uptake via AMPK is mediated by several factors, such as the increase in intracellular concentrations $\mathrm{Ca}^{2+}$ and bradykinin, also by the increase in the activity of nitric oxide synthase endothelial, by the use of the protein kinase activated by mitoxide (MAPK), activation of $\mathrm{Ca}^{2+} /$ calmodulin-dependent protein kinase $(\mathrm{CaMK})$ and activation of protein kinase $\mathrm{C}$ (PKC), in addition to hypoxia condition ${ }^{46}$. Thus, RT reduces glucose levels as well as lipid profile, as observed in our study. Besides, it is worth remembering that both hyperglycemia and hyperlipidemia are responsible for increasing the production of ROS and it can accelerate some pathogenesis related to obesity such as type 2 diabetes, as evidenced by glucose and lipid toxicity theories ${ }^{47}$. That is why controlling blood glucose, lipid profile, and improving the antioxidant system is so important, which can be done by RT. About the lipid profile, the same diet of the present experiments used for three weeks ${ }^{21}$ showed that such a period is enough to change the blood lipid profile and increase the mass of adipose tissue developing dyslipidemia.

Our results showed that the high-fat diet was responsible for the dyslipidemia condition, high levels of total cholesterol, VLDL-c, TGL, and even decrease of HDL-c. This condition is observed in rodent models of induced obesity ${ }^{20,34,48}$. Dobrian and co-workers ${ }^{35}$ studying the occurrence of hypertension in rats fed with a high-fat diet addressed an increase of LDL-c concentration and decreased HDL-c concentration after 10 weeks of intervention. Regarding the LDL-c, our results are not in agreement with the literature, maybe due to the dietary intervention span. Moreover, eight weeks of high-fat diet brought up early metabolic disorder, suggesting dyslipidemic conditions. This set of responses from the short trial span must be more investigated. Even so, RT in the same period was able to improve such dyslipidemic conditions by lowering lipid disorder parameters, enhancing HDL-c, and lessening the cardio-metabolic risk biomarkers such as the Castelli's ratio I and II, in either eutrophic or obese animals. Not only the whole organism could be affected by the negative effects of obesity but also the heart, a structure with intense metabolic activity, is prone to be affected by the obese condition ${ }^{49}$.

In the present study, an increase in LV lipid content was observed only in the trained obese group (Ob-RT). This was likely for the preference of carbohydrates (glucose) as the energy source instead of lipids, which were stored in the peripheral tissues, as just reported in skeletal muscle ${ }^{50}$. This increase of lipids associated with increased metabolic demand due to exercise elevates the work of cardiomyocytes. Thus, the energy needed by the cardiac tissue is augmented, followed by the heart mitochondria activity. This fact is corroborated by the increase of citrate synthase activity, as also observed in other study ${ }^{51}$. The growth of the total lipid content in LV of RT-Ob may be the consequence of the maximum capacity of the ventricular myocytes is reached by the oxidation of palmitate or other excessive free fatty acids stored in $\mathrm{LV}^{5}$. As observed, high-fat diets augmented the heart lipid concentration of Sprague Dawley ${ }^{5}$. However, improvement in oleate oxidation with consequent suppression of glucose oxidation has been reported in the cardiac tissue of rats fed with a diet rich in fat ${ }^{52}$. The differences between results are probably due to distinctions among animals and protocols.

It has been shown in rats fed with caloric diets, in comparison with standard ones, that the experimental span can increase the mitochondrial fatty acid oxidation causing damages in cardiac output without changes of citrate synthase activity. However, the oxygen uptake in vivo is $19 \%$ higher and the cardiac efficiency is $21 \%$ lower due to the increase in oxygen consumption $^{53}$. In contrast to the lower citrate synthase activity up discussed, the higher activity usually observed in animals submitted to RT protocols is being assumed to be due to the lipid accumulation in the LV and the increased metabolic demand from the imposed exercise. Moreover, RT improves cardiac efficiency reducing blood pressure ${ }^{20,54-56}$. The increment of energy production by cardiac mitochondria comes from fatty acids catabolism as the predominant metabolic resource. However, high mitochondrial work can generate more superoxide radicals in the cardiomyocytes ${ }^{57}$.

The activity of antioxidant enzymes is usually increased with the rise of superoxide anions, which can lead to damages particularly in the heart tissue. As a protective factor, the body makes use of the first line of antioxidant defenses, SOD, and CAT activities to cope with superoxides. The induced obesity in the Wistar rats does not modify the action of the antioxidant defense system. However, the high values of the Total-SOD 
activity in the RT group allowed us to assume that RT produced superoxide radicals in LV. Even obese rats depicted the same effects when submitted to RT. In obese rats submitted to RT, however, the increase of antioxidant response was observed through Mn-SOD and CAT activities. The effectiveness of the RT protocol has been shown to improve the heart antioxidant defenses ${ }^{18,58}$. Comparison between sedentary Fisher rats with others submitted for eight weeks to the aerobic protocol in a treadmill shows a greater activity of Total-SOD, Mn-SOD, CAT, and GSH in the trained rats. This response is indicative of cardioprotective effects from the aerobic exercise to cope with the oxidative stress ${ }^{59}$.

Observing the effect of strength training, under submaximal for short period (one month) or long period (three months) on cardiac function in rats, is found that the developed an increase of the cardiac mass and the relative heart mass. The heart rate baseline is lower in animals trained for three months, and coronary blood flow is increased either in sedentary or exercised rats. There is no change in the enzymatic activity of SOD, CAT, GPx, and lipid peroxidation either in trained or sedentary animals, allowing concluding that sub-maximal intensity is appropriate and safe for the heart tissue ${ }^{18}$. The results of our study differ from those cited here, probably due to the different protocols applied. Juvenile rats fed with high-fat diet depict a significant increase of BM, heart rate, visceral adiposity, plasma lipid peroxidation, and blood pressure ${ }^{60}$. Obese animals presented an increase in intravascular volume and cardiac output. This fact is due to the need for matching the metabolic demand with the $\mathrm{BM}$, particularly in adipose tissue, the total peripheral resistance, and the sympathetic activity ${ }^{61,62}$.

It was observed an increased activity of the Active MMP-2 in the LV of rats fed with high-fat diets. This response likely built up the proteolytic action of ECM constituents such as collagen I and IV, and LV sarcomere proteins such as troponin I, myosin light head I due to the action of Pro MMP- $2^{15}$. However, the lower activity of Pro and Intermediate MMP-2 here observed may be related to the activity of the Active MMP-2. Those effects could lead to a minor cardiac function, making it necessary for enhancing the heart work to provide nutrients to the entire organism through the vascular system. To reach these conditions it is necessary elevating the heart energy production which will lead to elevated citrate synthase activity, $\mathrm{O}_{2}$ - production, activation of MMP-2, and elevated antioxidant work, such as observed in the present study.

The increase of lipid peroxidation results in MMP-2 activity, remodeling the LV either through Pro (with its pro-domain, activated by nitric peroxide and GSH) or active isoforms ${ }^{14}$. It is postulated that the post-translational modification of MMP$2,72 \mathrm{kDa}$ (Pro MMP-2) by oxidative stress is a key event that leads to its intracellular activation observed in several pathological conditions, including obesity ${ }^{10}$. These facts support our assumptions. The obesity-induction period was able to promote alterations in the lipid profile, oxidative stress, and increased activity of MMP-2 in the LV. However, RT partially inhibited this activity, showing lower values in obese animals and eutrophic ones, as observed and previously reported ${ }^{17}$.

Reviews on MMP-2 have shown that this enzyme, activated by oxidative stress and proteolytic actions, is also associated with apoptosis by stimulation of $\beta$-adrenergic receptors. The MMP-2 active, along with TNF- $\alpha$ induces apoptosis of cardiac cells. There is evidence of the role of both in apoptotic pathways of myocardial cells and other cellular functions, either over healthful or pathogenic condition ${ }^{14}$. The degradation of contractile proteins as signaling to apoptosis by MMP-2 is related to oxidative stress in cardiomyocytes ${ }^{10,14,15}$. Thus, decreasing the MMP-2 activity could protect cardiac tissue from the harmful effects of oxidative stress-induced by obesity. In our study, the eight weeks of RT was able to promote a decrease of MMP-2 activity in obesity-induced by diet. This finding points out to a beneficial response of RT protocol with outcomes on mitigating deleterious effects from obesity and possible improvement of cardiac functions from such pathological conditions.

In summary, eight weeks of high-fat diet promotes an elevated body mass gain, and increase obesity index, accompanied by an alteration in the lipid profile. Therefore, RT was able to prevent body weight gain, to reduce the obesity index and to improve the lipid profile, to elevate the activation of the citrate synthase, and to decrease MMP-2 activity in the LV of rats.

\section{Conclusion}

In the current study, eight weeks of RT in obese animals was efficient in reversing the harmful effects of obesity in dyslipidemic condition, ameliorating lipidic profile, and cardiometabolic parameters and equilibrating MMP-2 activity in LV of obese rats. Besides that, obesity-induced by high-fat diet leads to metabolic lipid alterations, the elevation of lipid content, an increase of citrate synthase activity, and elevated the MMP-2 activity.

\section{References}

1. Apovian CM. Obesity: definition, comorbidities, causes, and burden. Am J Manag Care. 2016;22(7 Suppl):s176-85.

2. World Health Organization, Obesity and overweight2016 June, 2016. Available from: http:/www.who.int/mediacentre/factsheets/fs311/en/ [Accessed 18th October 2016].

3. Swinburn BA, Caterson I, Seidell JC, James WP. Diet, nutrition and the prevention of excess weight gain and obesity. Public Health Nutr. 2004;7(1A):123-46.

4. Wang W, Schulze CJ, Suarez-Pinzon WL, Dyck JR, Sawicki G, Schulz R. Intracellular action of matrix metalloproteinase-2 accounts for acute myocardial ischemia and reperfusion injury. Circulation. 2002;106(12):1543-9.

5. Akki A, Seymour AM. Western diet impairs metabolic remodelling and contractile efficiency in cardiac hypertrophy. Cardiovasc Res. 2009;81(3):610-7.

6. de Simone G, Pasanisi F, Ferrara AL, Roman MJ, Lee ET, Contaldo F, et al. Relative fat-free mass deficiency and left ventricular adaptation to obesity: The Strong Heart Study. Int J Cardiol. 2012 168(2):729-33.

7. Derosa G, Ferrari I, D'Angelo A, Tinelli C, Salvadeo SA, Ciccarelli L, et al. Matrix metalloproteinase-2 and -9 levels in obese patients. Endothelium. 2008;15(4):219-24. 
8. Droge W. Free radicals in the physiological control of cell function. Physiol Rev. 2002;82(1):47-95.

9. Noeman SA, Hamooda HE, Baalash AA. Biochemical study of oxidative stress markers in the liver, kidney and heart of high-fat diet induced obesity in rats. Diabetol Metab Syndr. 2011;3(1):17.

10. Viappiani S, Nicolescu AC, Holt A, Sawicki G, Crawford BD, Leon $\mathrm{H}$, et al. Activation and modulation of $72 \mathrm{kDa}$ matrix metalloproteinase- 2 by peroxynitrite and glutathione. Biochem Pharmacol. 2009;77(5):826-34.

11. Hadler-Olsen E, Fadnes B, Sylte I, Uhlin-Hansen L, Winberg $\mathrm{J}-\mathrm{O}$. Regulation of matrix metalloproteinase activity in health and disease. FEBS Journal. 2011;278(1):28-45.

12. Kehat I, Molkentin JD. Molecular pathways underlying cardiac remodeling during pathophysiological stimulation. Circulation. 2010;122(25):2727-35.

13. Sanchez-Pozo J, Baker-Williams AJ, Woodford MR, Bullard R, Wei B, Mollapour M, et al. Extracellular Phosphorylation of TIMP-2 by Secreted c-Src Tyrosine Kinase Controls MMP-2 Activity. iScience. 2018;1:87-96.

14. Kandasamy AD, Chow AK, Ali MA, Schulz R. Matrix metalloproteinase-2 and myocardial oxidative stress injury: beyond the matrix. Cardiovasc Res. 2010;85(3):413-23.

15. Chow AK, Cena J, Schulz R. Acute actions and novel targets of matrix metalloproteinases in the heart and vasculature. $\mathrm{Br} \mathrm{J}$ Pharmacol. 2007;152(2):189-205.

16. Xian X, Ma Y, Yang DD, Huang W, Wang Y, Mueller O, et al. Reduced high-density lipoprotein $2 \mathrm{~b}$ in non-obese type 2 diabetic patients analysed by a microfluidic chip method in a case-control study. Biomarkers. 2009;14(8):619-23.

17. Leite RD, Durigan RCM, Lino ADS, Campos MVS, Souza MG, Selistre-de-Araújo HS, et al. Resistance Training may concomitantly benefit body composition, blood pressure and muscle MMP-2 activity on the left ventricle of high-fat fed diet rats. Metab Clin Exp. 2013.

18. Ahmadiasl N, Najafipour H, Soufi FG, Jafari A. Effect of shortand long-term strength exercise on cardiac oxidative stress and performance in rat. J Physiol Biochem. 2012;68(1):121-8.

19. Marqueti RC, Micocci KC, Leite RD, Selistre-de-Araujo HS. Nandrolone inhibits MMP-2 in the left ventricle of rats. Int $\mathrm{J}$ Sports Med. 2012;33(3):181-5.

20. Leite RD, Durigan RC, Lino ADS, Campos MVS, Souza M, Selistre-de-Araujo HS, et al. Resistance training may concomitantly benefit body composition, blood pressure and muscle MMP-2 activity on the left ventricle of high-fat fed diet rats. Metab Clin Exp. 2013;62(10):1477-84.

21. Estadella D, Oyama LM, Dâmaso AR, Ribeiro EB, Oller Do Nascimento CM. Effect of palatable hyperlipidic diet on lipid metabolism of sedentary and exercised rats. Nutrition 2004(20):218-24.

22. Hornberger TA, Jr., Farrar RP. Physiological hypertrophy of the FHL muscle following 8 weeks of progressive resistance exercise in the rat. Can J Appl Physiol. 2004;29(1):16-31.

23. Leite RD, Prestes J, Bernardes CF, Shiguemoto GE, Pereira GB, Duarte JO, et al. Effects of ovariectomy and resistance training on lipid content in skeletal muscle, liver, and heart; fat depots; and lipid profile. Appl Physiol Nutr Metab. 2009;34(6):1079-86.
24. Speretta GF, Rosante MC, Duarte FO, Leite RD, Lino AD, Andre RA, et al. The effects of exercise modalities on adiposity in obese rats. Clinics (Sao Paulo). 2012;67(12):1469-77.

25. Cinti S. The adipose organ. Prost Leukot Esse Fat Acids. 2005;73(1):9-15.

26. D'Eon TM, Souza SC, Aronovitz M, Obin MS, Fried SK, Greenberg AS. Estrogen regulation of adiposity and fuel partitioning. Evidence of genomic and non-genomic regulation of lipogenic and oxidative pathways. J Biol Chem. 2005;280(43):35983-91.

27. Friedewald WT, Levy RI, Fredrickson DS. Estimation of the concentration of low-density lipoprotein cholesterol in plasma, without use of the preparative ultracentrifuge. Clin Chem. 1972;18(6):499-502.

28. Castelli WP, Abbott RD, McNamara PM. Summary estimates of cholesterol used to predict coronary heart disease. Circulation. 1983;67(4):730-4.

29. Beutler E. Red cell metabolism: a manual of biochemical methods. 3rd Stratton G, editor. New York: Grune \& Stratton, 1984.

30. Kruger N. The Bradford method for protein quantifica- tion. Methods Mol Biol. 1994;32:9-15.

31. Frings CS, Fendley TW, Dunn RT, Queen CA. Improved determination of total serum lipids by the sulfo-phospho-vanillin reaction. Clin Chem. 1972;18(7):673-4.

32. Sene-Fiorese M, Duarte FO, Scarmagnani FR, Cheik NC, Manzoni MS, Nonaka KO, et al. Efficiency of intermittent exercise on adiposity and fatty liver in rats fed with high-fat diet. Obesity (Silver Spring). 2008;16(10):2217-22.

33. Cao Y. Adipose tissue angiogenesis as a therapeutic target for obesity and metabolic diseases. Nat Rev Drug Discov. 2012; 9(2):107-15.34.

34. Duarte FO, Sene-Fiorese M, Cheik NC, Maria AS, de Aquino AE, Jr., Oishi JC, et al. Food restriction and refeeding induces changes in lipid pathways and fat deposition in the adipose and hepatic tissues in rats with diet-induced obesity. Exp Physiol. 2012;97(7):882-94.

35. Dobrian AD, Davies MJ, Prewitt RL, Lauterio TJ. Development of hypertension in a rat model of diet-induced obesity. Hypertension. 2000;35(4):1009-15.

36. Campbell PT, Gross MD, Potter JD, Schmitz KH, Duggan C, McTiernan A, et al. Effect of Exercise on Oxidative Stress. Med Sci Sports Exerc. 2010; 42(8):1448-53.

37. Stenholm S, Alley D, Bandinelli S, Griswold ME, Koskinen S, Rantanen $\mathrm{T}$, et al. The effect of obesity combined with low muscle strength on decline in mobility in older persons: results from the InCHIANTI study. Int J Obes (Lond). 2009;33(6):635-44.

38. Hulens M, Vansant G, Lysens R, Claessens AL, Muls E, Brumagne S. Study of differences in peripheral muscle strength of lean versus obese women: an allometric approach. Int J Obes Relat Metab Disord. 2001;25(5):676-81.

39. Maffiuletti NA, Jubeau M, Munzinger U, Bizzini M, Agosti F, De Col A, et al. Differences in quadriceps muscle strength and fatigue between lean and obese subjects. Eur J Appl Physiol. 2007;101(1):51-9.

40. Willis LH, Slentz CA, Bateman LA, Shields AT, Piner LW, Bales $\mathrm{CW}$, et al. Effects of aerobic and/or resistance training on body mass and fat mass in overweight or obese adults. J Appl Physiol. 2012;113(12):1831-7. 
41. Saengsirisuwan V, Kinnick TR, Schmit MB, Henriksen EJ. Interactions of exercise training and lipoic acid on skeletal muscle glucose transport in obese Zucker rats. J Appl Physiol. 2001;91(1):145-53.

42. Tzagkaroulakis A, Stivaktakis J, Nikolopoulos T, Davilis D, Zervoudakis D. Ancient schwannoma of the true vocal cord. ORL J Otorhinolaryngol Relat Spec. 2003;65(5):310-3.

43. Maarbjerg SJ, Sylow L, Richter EA. Current understanding of increased insulin sensitivity after exercise - emerging candidates. Acta Physiol. 2011;202(3):323-35.

44. Saengsirisuwan V, Perez FR, Sloniger JA, Maier T, Henriksen EJ. Interactions of exercise training and alpha-lipoic acid on insulin signaling in skeletal muscle of obese Zucker rats. Am J Physiol Endocrinol Metab. 2004;287(3):E529-36.

45. Krisan AD, Collins DE, Crain AM, Kwong CC, Singh MK, Bernard JR, et al. Resistance training enhances components of the insulin signaling cascade in normal and high-fat-fed rodent skeletal muscle. J Appl Physiol. 2004;96(5):1691-700.

46. Ferrari F, Bock PM, Motta MT, Helal L. Biochemical and Molecular Mechanisms of Glucose Uptake Stimulated by Physical Exercise in Insulin Resistance State: Role of Inflammation. Arq Bras Cardiol. 2019;113(6):1139-48.

47. Chang YC, Chuang LM. The role of oxidative stress in the pathogenesis of type 2 diabetes: from molecular mechanism to clinical implication. Am J Transl Res. 2010;2(3):316-31.

48. Estadella D, Oyama LM, Dâmaso AR, Ribeiro EB, Oller Do Nascimento CM. Effect of palatable hyperlipidic diet on lipid metabolism of sedentary and exercised rats. Nutrition. 2004;20(2):218-24.

49. Van Gaal LF, Mertens IL, De Block CE. Mechanisms linking obesity with cardiovascular disease. Nature. 2006;444(7121):875-80.

50. Hegarty BD, Cooney GJ, Kraegen EW, Furler SM. Increased efficiency of fatty acid uptake contributes to lipid accumulation in skeletal muscle of high-fat-fed insulin-resistant rats. Diabetes. 2002;51(5):1477-84.

51. Boudina S, Sena S, O’Neill BT, Tathireddy P, Young ME, Abel ED. Reduced mitochondrial oxidative capacity and increased mitochondrial uncoupling impair myocardial energetics in obesity. Circulation. 2005;112(17):2686-95.

52. Wilson CR, Tran MK, Salazar KL, Young ME, Taegtmeyer H. Western diet, but not high-fat diet, causes derangements of fatty acid metabolism and contractile dysfunction in the heart of Wistar rats. Biochem J. 2007;406(3):457-67.

53. Cole MA, Murray AJ, Cochlin LE, Heather LC, McAleese S, Knight NS, et al. A high-fat diet increases mitochondrial fatty acid oxidation and uncoupling to decrease efficiency in rat heart. Basic Res Cardiol. 2011;106(3):447-57.

54. Pescatello LS, Franklin BA, Fagard R, Farquhar WB, Kelley GA, Ray CA, et al. American College of Sports Medicine position stand. Exercise and hypertension. Med Sci Sports Exerc. 2004;36(3):533-53.

55. Moraes MR, Bacurau RF, Simoes HG, Campbell CS, Pudo MA, Wasinski F, et al. Effect of 12 weeks of resistance exercise on post-exercise hypotension in stage 1 hypertensive individuals. J Hum Hypertens. 2012;26(9):533-9.
56. Westcott WL. Resistance training is medicine: effects of strength training on health. Curr Sports Med Rep. 2012;11(4):209-16.

57. Powers SK, Jackson MJ. Exercise-induced oxidative stress: cellular mechanisms and impact on muscle force production. Physiol Rev. 2008;88(4):1243-76.

58. Williams MA, Haskell WL, Ades PA, Amsterdam EA, Bittner V, Franklin BA, et al. Resistance Exercise in Individuals With and Without Cardiovascular Disease: 2007 Update: A Scientific Statement From the American Heart Association Council on Clinical Cardiology and Council on Nutrition, Physical Activity, and Metabolism. Circulation. 2007;116(5):572-84.

59. Husain K, Hazelrigg SR. Oxidative injury due to chronic nitric oxide synthase inhibition in rat: effect of regular exercise on the heart. Biochim Biophys Acta. 2002;1587(1):75-82.

60. Smith AD, Brands MW, Wang MH, Dorrance AM. Obesityinduced hypertension develops in young rats independently of the renin-angiotensin-aldosterone system. Exp Biol Med. 2006;231(3):282-7.

61. Blake J, Devereux RB, Borer JS, Szulc M, Pappas TW, Laragh JH. Relation of obesity, high sodium intake, and eccentric left ventricular hypertrophy to left ventricular exercise dysfunction in essential hypertension. Am J Med. 1990;88(5):477-85.

62. Reisin E, Jack AV. Obesity and hypertension: mechanisms, cardio-renal consequences, and therapeutic approaches. Med Clin North Am. 2009;93(3):733-51.

\section{Acknowledgments}

The authors are grateful to Mr. José Alves da Silva, Fernanda Moraes, Rita de Cassia Marqueti Durigan and Anabelle Silva Cornachione for technical laboratory assistance. Financial support was provided by the Consellho Nacional de Desenvolvimento Científico e Tecnológico (CNPq), Brazil.

\section{Corresponding author}

Anderson Diogo de Souza Lino

Programa de Pós-Graduação em Educação Física (PPGEF), Av. do Tutuna, $420-$ Vila Celeste, Uberaba, MG, CEP: 38061-500 - +55 (34) 9 9209-2543 Email: andersonlino@hotmail.com

Associate Editor: Fernanda B. M. Priviero. Augusta University, US.

Manuscript received on November 4, 2019

Manuscript accepted on July 10, 2020

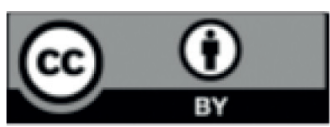

Motriz. The Journal of Physical Education. UNESP. Rio Claro, SP, Brazil - eISSN: 1980-6574 - under a license Creative Commons - Version 4.0 